\title{
3-D modeling of the collimated outflows of M1-32 and M2-42
}

\author{
Stavros Akras, Jose-Alberto López and Wolfgang Steffen
}

Instituto de Astronomia Universidad National Autónoma de Mexico, Ensenada, Mexico

email: akras@astrosen. unam.mx

email: jal@astrosen.unam.mx

email: wsteffen@astrosen. unam.mx

\begin{abstract}
We present high resolution long-slit spectra from the San Pedro Martir kinematic catalogue (López et al. 2012) of plantetary nebulae M1-32 and M2-42 and their modeled PV diagrams using the 3-D morpho-kinematic code SHAPE. We find high-speed collimated outflows, surrounded by a equatorial thick torus/ring. The same SHAPE model is able to fit both planetary nebulae assuming one cylindrical velocity field for the outflows and one Hubble-law for the torus/ring concluding that they may be akin objects which just appear at different projections.
\end{abstract}

Keywords. ISM: jets and outflows, ISM: kinematics and dynamics, methods: numerical, planetary nebula: individual (M1-32, M2-42)

\section{Introduction}

Many surveys have been made to discover as many as possible Planetary nebulae (PNe) (see Parker \& Frew 2010) revealing a variety of complex morphologies. Based on the theoretical models, the complexity of PNe structure occur due to the presence of magnetic field (Garcia-Segura 1997), the binarity of central star (CS) (Balick \& Frank 2002), the photo-ionization heating or the rotation of the central star (Garcia-Segura et al. 1999). Spectroscopic and imaging information are used in order to reconstruct a 3-D structure of M1-32 and M2-42, making a usage of the 3-D morpho-kinematic code SHAPE (Steffen et al. 2011).

\section{Observations}

High resolution spectra of M1-32 \& M2-42 were obtained with $2.1 \mathrm{~m}$ telescope at San Pedro Martir Observatory in Baja California, Mexico with the Manchester echelle spectrometer (MES-SPM) in June 2007 and in July 2009 (The SPM Kinemtatic Catalogue
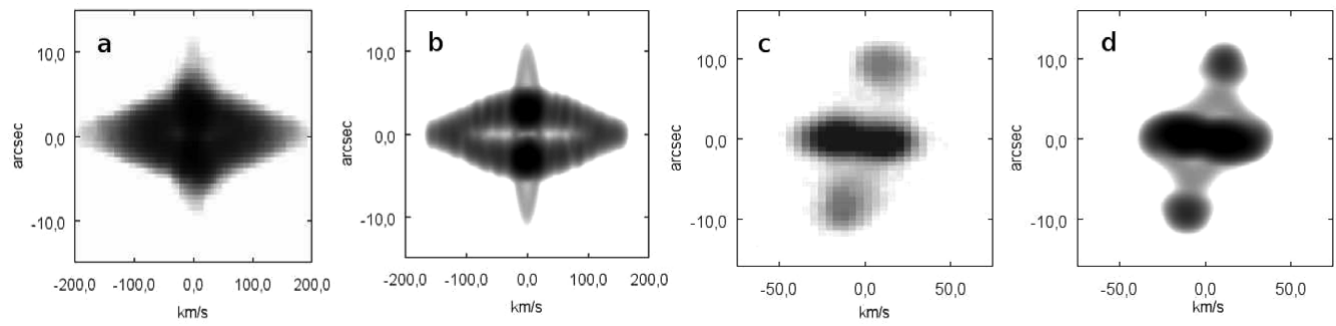

Figure 1. The observed and modeled synthetic PV diagrams of [N II] emission line of M1-32 (a \& b) and M2-42 (c \& d). 

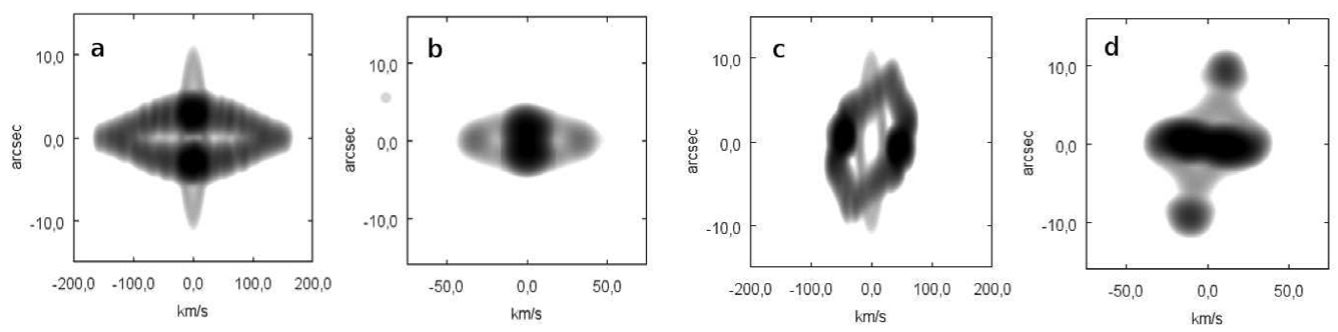

Figure 2. The synthetic PV diagrams of M 1-32 (a) \& M 2-42 (b) at 0 degree inclination angle (pole-on). The corresponding synthetic PV diagrams of M1-32 (c) and M2-42 (b) at 90 degrees inclination angle.

of Planetary Nebulae (López et al. 2012). The slit width was $1.9 \operatorname{arcsec}(150 \mathrm{~m})$ for a spectra resolution equivalent to $11 \mathrm{~km} / \mathrm{s}$. The observed $\mathrm{PV}$ diagrams are presented in Figure 1.

\section{SHAPE modeling}

In order to understand better the nature of the highly collimated outflows of M1$32 \&$ M2-42, we use the morpho-kinematic code SHAPE and their PV diagrams from the San Pedro Martir kinematic catalogue. Both nebula show highly collimated outflows surrounded by a equatorial thick torus/ring. A cylindrical velocity field is needed in order to produce the high moving collimated outflows along the polar direction. In addition, a Hubble law is used for the torus/ring component. Hence, the same SHAPE model is able to replicate the observation for both nebulae. The final synthetic PV diagrams are shown in Figures 1 and 2 with the corresponding observed PV diagram. A similar cylindrical velocity field is used to produce the outflows of the young planetary nebula $\mathrm{BD}+30^{\circ} 3639$. (Akras \& Steffen, theses proceedings)

\section{Conclusions}

1) A cylindrical velocity component, increasing linearly with the distance from the central star, can adequately reproduce the high collimated biporar outlows. 2) The equatorial thick torus/ring expands radially following a Hubble-law. 3) The most significant difference between M1-32 and M2-42 is that the former shows larger polar and equatorial expansion velocities. 4) According to our modeled PV diagrams, we find that both $\mathrm{PNe}$ are akin objects seemingly formed by similar mechanisms and just appear at different projections on the sky. Consequently, the same model fits both Planetary Nebulae (fig. 3). 5) We also find that the collimared outflows are formed by the similar mechanish either for PNe with WR central star (M1-32) or weak emission lines (wels) central star (M2-42).

\section{References}

Balick, B. \& Frank, A. 2002, ARA\&A, 40, 439

Garcia-Segura, G. 1997, ApJ, 489, 189

Garcia-Segura, G., Langer, N., Różyczka, M., \& Franco, J. 1999, ApJ, 517, 767

López, J. A., Richer, M. G., García-Díaz, M. T., et al. 2012, RMxAA, 48, 3

Parker, Q. A. \& Frew, D. 2011, , arXiv1011.0857

Steffen, W., Koning, N., Wenger, S., Morisset, C., \& Magnor, M. 2011, Transactions of Computer Graphics and Visualization, 17, 454 